\title{
Section E6.5-6.8 of the ACMG technical standards and guidelines: chromosome studies of lymph node and solid tumor-acquired chromosomal abnormalities
}

\author{
Linda D. Cooley, MD, MBA 1 , Cynthia C. Morton, PhD²,3, Warren G. Sanger, PhD ${ }^{4,7}$, Debra F. Saxe, PhD ${ }^{5}$ \\ and Fady M. Mikhail, MD, PhD; ; on behalf of the American College of Medical Genetics and \\ Genomics (ACMG) Laboratory Quality Assurance Committee
}

\begin{abstract}
Disclaimer: These ACMG standards and guidelines are developed primarily as an educational resource for clinical laboratory geneticists to help them provide quality clinical laboratory genetic services. Adherence to these standards and guidelines is voluntary and does not necessarily ensure a successful medical outcome. These standards and guidelines should not be considered inclusive of all proper procedures and tests or exclusive of other procedures and tests that are reasonably directed to obtaining the same results. In determining the propriety of any specific procedure or test, the clinical laboratory geneticist should apply his or her own professional judgment to the specific circumstances presented by the individual patient or specimen. Clinical laboratory geneticists are encouraged to document in the patient's record the rationale for the use of a particular procedure or test, whether or not it is in conformance with these standards and guidelines. They also are advised to take notice of the date any particular guideline was adopted, and to consider other relevant medical and scientific information that becomes available after that date. It also would be prudent to consider whether intellectual property interests may restrict the performance of certain tests and other procedures.
\end{abstract}

Cytogenetic analysis of tumor tissue is performed to detect and characterize chromosomal aberrations to aid histopathological and clinical diagnosis and patient management. At the time of diagnosis, known recurrent clonal aberrations may facilitate histopathological diagnosis and subtyping of the tumor. This information may contribute to clinical therapeutic decisions. However, even when tumors have a known recurrent clonal aberration, each tumor is genetically unique and probably heterogeneous. It is important to discover as much about the genetics of a tumor at diagnosis as is possible with the methods available for study of the tumor material. The information gathered at initial study will inform follow-up studies, whether for residual disease detection, determination of relapse and clonal evolution, or identifying a new disease clone.
This updated Section E6.5-6.8 has been incorporated into and supersedes the previous Sections E6.4 and E6.5 in Section E: Clinical Cytogenetics of the 2009 Edition (Revised 01/2010), American College of Medical Genetics and Genomics Standards and Guidelines for Clinical Genetics Laboratories. This section deals specifically with the standards and guidelines applicable to lymph node and solid tumor chromosome analysis.

Genet Med advance online publication 28 April 2016

Key Words: cancer cytogenetics; chromosome; guidelines; lymph node; solid tumor

\subsection{GENERAL CONSIDERATIONS}

6.5.1 Genetic analysis of solid tumors and lymphomas at diagnosis provides information critical for diagnosis and patient management. ${ }^{1,2}$ Analysis of tumor tissues may be accomplished by conventional chromosome analysis, fluorescence in situ hybridization (FISH) analysis, chromosomal microarray (CMA) analysis, molecular analysis, or a combination of methodologies. Because the genetic information aids in the differential diagnosis and provides direction for the most appropriate therapeutic management, including targeted therapies, tumor materials should be studied with available methods to gain as much information as possible at the time of initial study. At a time of suspected disease recurrence or metastasis, the initial genetic data will be used to confirm recurrence or metastasis, assess clonal disease evolution, or reveal a new malignant process.

The method(s) chosen for evaluation of a tumor at the time of biopsy or resection will depend on the differential diagnosis, clinical indications, available tissue, available methodologies, and initial histopathology of the tumor tissue.

For disease staging, tumor samples may be accompanied or followed by other tissue samples for analysis, such as bone marrow and cerebrospinal fluid.

\footnotetext{
${ }^{1}$ Department of Pathology \& Laboratory Medicine, Children's Mercy Hospital, University of Missouri Kansas City Medical School, Kansas City, Missouri, USA; ${ }^{2}$ Department of Obstetrics, Gynecology and Reproductive Biology, Brigham and Women's Hospital, Harvard Medical School, Boston, Massachusetts, USA; ${ }^{3}$ Department of Pathology, Brigham and Women's Hospital, Harvard Medical School, Boston, Massachusetts, USA; ${ }^{4}$ Department of Pathology, University of Nebraska Medical Center, Omaha, Nebraska, USA; ${ }^{5}$ Department of Pathology, Emory University School of Medicine, Atlanta, Georgia, USA; ${ }^{6}$ Department of Genetics, University of Alabama at Birmingham, Birmingham, Alabama, USA. ${ }^{7}$ Deceased. Correspondence: Linda D. Cooley (lcooley@cmh.edu)
}

Approved by the ACMG Board of Directors on 25 January 2016. 
6.5.2 The laboratory director and staff should be familiar with the chromosomal and molecular aberrations associated with tumor types/subtypes and their clinical significance. Supplementary Tables S1-S5 online include common solid tumor and lymphoma chromosomal aberrations with known genes, potential FISH targets, clinical significance, and references.

6.5.3 Pediatric tumors should be cytogenetically analyzed whenever sufficient fresh tissue is available. Karyotyping, although low-resolution, provides a view of the entire genome. This genome view allows detection of cytogenetic aberrations that are commonly disease- or disease subtype-specific and have prognostic and therapeutic significance. Genetic analysis of adult tumors is indicated whenever such analysis may provide diagnostic, prognostic, or treatment-related information, especially if targeted therapies are available for the disorder undergoing study.

6.5.4 Methods for the processing of tumor material should be determined by the cytogenetic laboratory based on available clinical and pathologic findings. Laboratories should work with the oncologist and pathologist to determine the method(s) to gain the most genetic information cost-effectively. The laboratory should seek information about the suspected diagnosis and tissue type at the time of sample receipt to choose the most appropriate testing and tissue culture method(s) and to determine if DNA should be isolated from the fresh tumor. Supplementary Table S6 online provides tumor nomenclature for tumor culture method selection.

6.5.5 Conventional cytogenetic, FISH, CMA, gene mutation panel, or sequencing analysis may be used as a primary or secondary method of evaluation of the tumor tissue. Multiple technologies may be needed for specific tumor types. The availability of fresh tissue, the differential diagnosis, a need for rapid diagnostic information, and the type of information needed should be used to prioritize testing such as conventional cytogenetic analysis, FISH, CMA, and/or mutation analysis.

6.5.6 Cytogenetic and molecular analysis results must be interpreted within the context of the pathologic and clinical findings.

6.5.7 For quality assurance, the laboratory may monitor the number and types of tumors received, the percentage of tumors with abnormal results, the cell culture success rate, and the success rate for FISH and CMA studies.

6.5.8 The presence or absence of specific aberrations should be available to the physician as soon as is feasible to contribute to the patient's plan of care.

\subsection{SAMPLE COLLECTION AND PROCESSING 6.6.1 Sample collection}

6.6.1.1 Tumor samples should be collected in a sterile manner. For conventional cytogenetic analysis, the tissue sample must be fresh. The sample selected for cytogenetic analysis should be "pure" tumor if possible, without necrosis. The sample must not be placed in fixative or frozen. Samples to be evaluated solely by FISH or CMA analysis may be fixed, frozen, or paraffinembedded. If CMA analysis or sequencing is requested at the time of biopsy, DNA should be isolated from fresh tumor or formalin-fixed paraffin-embedded tumor rather than cultured tumor cells because clonal aberrations may be lost during cell culture. Cultured tumor cells may be used for isolation of DNA if the karyotype is clonally abnormal. The use of formalin-fixed paraffin-embedded samples for FISH and DNA isolation allows a pathologist to identify and mark optimal areas of tumor to examine, specify the percentage of tumor in an area, and/or identify areas of necrosis or stromal tissue to avoid.

6.6.1.2 The laboratory should request a sample size of 0.5 to $1 \mathrm{~cm}^{3}$. If less tissue is available, the laboratory should accept as much as can be provided. If the sample size is very limited (e.g., fine needle aspirate or needle core biopsy), coverslip cultures are often successful. If the sample size precludes cell culture and conventional cytogenetic evaluation, touch preparations, cytospins, or paraffin-embedded tissue sections may be used for FISH analysis, or DNA may be isolated for CMA or sequencing analysis. See Section E6.5.2.

6.6.1.3 Fresh tumor should be transported in culture medium to the cytogenetics laboratory as soon as possible for immediate processing.

\subsubsection{Sample processing}

6.6.2.1 The cytogenetic laboratory should process the tumor sample as soon as possible after it is received. Prior to processing, it should be clear what methods will be used to analyze the sample (e.g., chromosome analysis, FISH, CMA, sequencing). If the sample is to be processed for CMA or sequencing, select a portion of the sample for DNA isolation. If the sample is for FISH analysis, touch preparations may be made or direct harvest performed. If the sample is for chromosome analysis, tissue culture will be required.

6.6.2.2 The fresh tumor sample should be inspected and details of the sample size, color, and attributes recorded. The time of sample collection and the time of sample receipt in the laboratory should be documented.

6.6.2.3 The cytogenetics laboratory should expect the sample submitted by a pathologist to be most representative of the tumor as determined by gross examination. However, if the fresh sample received by the laboratory is large and appears heterogeneous, portions of the sample may be cultured separately. If obvious normal, necrotic, or vascular tissues are present, the tumor should be separated from nontumor tissue for processing. Obvious necrotic tissue should be removed to reduce enzymatic damage induced by dying cells. If the tumor cannot be distinguished from normal or necrotic tissue, caution should be exercised and the entire sample processed.

6.6.2.4 For tissues from a body region with high concentrations of bacteria (e.g., tonsils, gut), treatment of the sample prior to disaggregation with antibiotic and/or antifungal solutions and addition of antibiotic and/or antifungals to the medium may be prudent.

6.6.2.5 Disaggregation methods should be optimized for different tissue types:

a. Disaggregation of solid tumor samples for tissue culture is needed. Mechanical and/or enzymatic methods may 
be used. If sufficient tumor material is submitted, both methods of disaggregation are recommended. For some tumor types, different growth characteristics can be seen with exposure to collagenase versus no exposure to collagenase. If sufficient material is available, cultures should be initiated with and without enzyme exposure.

b. Disaggregation of lymphoid tissues into single cell suspension is necessary before culture initiation. The lymphoid cells in most tissues are readily disaggregated by mechanical means such as mincing with scalpels or curved scissors. The use of these methods is often advantageous if the tissue is easily dissociated because it will keep the loss of cells to a minimum and may help minimize stromal contamination because stromal cells are often locked in fibrous connective tissues. If cells are not readily liberated by mechanical means, enzymatic digestion may be necessary. When using enzymatic digestion, the tissue must first be minced and then incubated with the enzyme solution (e.g., collagenase) for 20 minutes to 16 hours depending on how quickly cell release occurs.

6.6.2.6 Culture methods, culture medium, and culture conditions should be chosen to best support the type of tumor received.

a. The diagnosis and histopathology of a tumor can be helpful in determining culture and harvest methods. Different cell types can be expected to respond differently with growth medium, harvest method, and other factors (Table 6). If the diagnosis is unknown at culture initiation, it can be helpful to know whether the pathologist would classify the tumor as a "small round cell tumor" (SRCT), which includes lymphoproliferative disorders. SRCTs can be successfully grown in suspension, whereas non-SRCTs are best grown with monolayer (flask or coverslip) culture methods. Most, but not all, SRCTs (e.g., lymphoproliferative disorders) will also grow in monolayer culture. If adequate tissue is obtained, both culture types should be initiated for SRCTs. For very small tumor samples, coverslip cultures are recommended. Duplicate cultures should be established whenever possible.

b. For lymphoid tissues, disaggregated cells are cultured in suspension using appropriate supportive growth medium. Tumor cells are spontaneously dividing; however, mitogens may be used for lymphoid disorders to encourage proliferation of the desired cell type.

6.6.2.7 Experience with solid tumor culture will provide the laboratory with information regarding optimal growth conditions and harvest methods for different tumor types.

a. It can be helpful for the laboratory to maintain a database that documents how the different tumor types have grown and which culture and harvest conditions yield abnormal clones. This database can then be searched for optimal processing and harvesting methods for any new tumor received in the laboratory.

b. Short culture durations are preferred to optimize the mitotic index of early dividing tumor cells and to avoid growth of normal tissues. Depending on the amount of available tissue, a combination of direct, 24-hour, and/ or 48-hour cultures are most often utilized for lymphoid disorders. Short-term cultures (e.g., direct or overnight cultures) may also be used in conjunction with longerterm cultures to capture actively dividing cells from solid tumors.

c. Frequent (daily) observation of cells in culture is needed to determine cell growth rate and optimal time to harvest. Tumor cells should be harvested as soon as possible upon adequate growth to capture early dividing tumor cells and to prevent overgrowth by chromosomally normal cells.

d. Conditions used for cell harvest will vary among tissue types (e.g., mitotic inhibitors) used (e.g., colcemid, velban, ethidium bromide), their concentration, and exposure duration, and they should be established by each laboratory.

\subsection{ANALYTICAL METHODS}

\subsubsection{Conventional G-banded chromosome analysis}

6.7.1.1 Cell selection. Analysis of metaphase chromosomes should include cells with both good and poor chromosome morphology when attempting to identify an abnormal clone. Once identified, clonal cells with the best chromosome morphology should be analyzed, karyotyped, and imaged to provide the most accurate breakpoint assignments.

Cells that cannot be completely analyzed because of poor morphology should be scanned for obvious structurally abnormal chromosomes and abnormal chromosome counts.

Clonal abnormalities should be documented in two independent cultures, if possible, to ensure that an in vitro culture artifact is not mistakenly identified as a clinically significant abnormality.

\subsubsection{Analytic standards}

\subsection{Initial diagnostic studies}

a. Analysis

i. Analyze 20 metaphase cells and/or a sufficient number of cells to characterize all abnormal clones and subclones.

ii. If all cells show a complex karyotype where each cell is different, then analyze at least 10 cells with karyotyping.

b. Documentation

i. For abnormal cells:

1. If only one abnormal clone is present: two karyotypes.

2. If more than one related abnormal clone is present: at least one karyotype of the stemline and at least one of each sideline. 
3. If unrelated clones are present: at least one karyotype for each stemline and one for each associated pertinent sideline.

ii. For normal cells:

1. If only normal cells are present: two karyotypes.

2. If normal and abnormal cells are present: one karyotype of a normal cell plus karyotypes for abnormal clone(s) as described.

6.7.1.2.2 Follow-up studies may be performed to assess stage of disease at the time of diagnosis or at the time of tumor recurrence.

a. Analysis

i. Analysis should include a minimum of 20 metaphase cells.

ii. Additional cells may be scored for a specific abnormality identified in the diagnostic sample.

iii. In addition to looking for the known clonal aberration(s) from the diagnostic study, analysis of a sample after therapy should be performed with awareness of the possibility of new aberrations signifying clonal evolution and/or a new clonal process (i.e., therapy-related malignancy).

iv. FISH analysis may be considered in lieu of conventional chromosomal analysis for diagnoses characterized by an abnormality for which FISH testing is available.

b. Documentation

i. If both normal and abnormal cells or if only abnormal cells are present:

1. One or two karyotypes from each abnormal clone with a minimum of two karyotypes.

2. One karyotype of a normal cell, if a normal karyotype was not documented in a previous study.

3. If only normal cells are present: two karyotypes.

\subsubsection{FISH analysis}

6.7.2.1 FISH analysis may be used for primary, supplementary, or follow-up evaluation

a. As a primary method for tumor evaluation, FISH is useful when (i) fresh tumor tissue is not available; (ii) rapid diagnostic information is needed to narrow the differential diagnosis; (iii) gene amplification or rearrangement for diagnostic or prognostic and/or therapeutic purposes is to be determined; (iv) no metaphase cells are obtained by culture of tumor material; or (v) conventional cytogenetic analysis yields a normal result.

b. Supplemental FISH may be used as an adjunct to the initial conventional chromosomal analysis or CMA analysis to: (i) document a specific molecular event (e.g., gene rearrangement or fusion); (ii) provide a rapid result to aid in the differential diagnosis or planning of therapy; (iii) to assess gene copy number,; (iv) clarify level of clonality; or (v) confirm a microarray variant. c. Follow-up FISH studies may be indicated to assess recurrent disease or disease progression and/or to differentiate recurrence of a tumor from a new disease process.

i. If initial studies failed to identify the clonal process unique to the tumor, then follow-up studies may provide another opportunity.

6.7.2.2 Characterization of interphase FISH aberrations and FISH signal patterns. Characterization of interphase FISH aberrations and the FISH signal patterns in diagnostic samples is useful for future monitoring of disease. Gene fusions may confirm a specific tumor diagnosis. If a particular patient's tumor has a unique FISH signal pattern, documentation of the pattern at diagnosis can prevent misinterpretation of FISH analysis at follow-up.

6.7.2.3 Sample types. Sample types that may be used for FISH include (i) paraffin-embedded tissue sections; (ii) touch preparations (TP); (iii) cytospin preparations; (iv) cultured or direct harvest tumor cells; (v) fixed cytogenetically prepared cells; or (vi) fresh-frozen tumor tissues.

a. Paraffin-embedded tissue ${ }^{3}$

i. Before scoring a paraffin-embedded FISH slide, it is crucial for a pathologist to review a hematoxylin and eosin-stained slide and delineate the region of tumor cells that should be scored because it can be difficult to differentiate normal cells from malignant cells using only DAPI counterstain. The technologist should be clear, before scoring the slide, where the malignant cells of interest are located on the slide.

ii. Formalin-fixed, paraffin-embedded tissue is acceptable for FISH analysis. Tissues preserved in B5 fixative or decalcified are not suitable for FISH.

iii. Tumor sections cut 3 to $4 \mu \mathrm{m}$ thick and mounted on positively charged organosilane-coated (silanized) slides work well. The cytogenetics laboratory should request several unstained sections and one hematoxylin and eosin-stained sequentially cut section from the submitting laboratory.

b. Touch preparations

i. A pathologist should make the TP or should be involved in selecting the tissue for TPs.

ii. TPs are helpful when tissue architecture is not crucial.

iii. TPs should be made by lightly touching the piece of tumor to a glass slide without smearing, followed by air drying.

c. Cytospin preparations

i. Cytospin preparations are useful for a concentration of samples with very low cellularity (e.g., cerebrospinal fluid).

d. Fixed cytogenetically prepared cells

i. Such preparations have multiple uses for both interphase and metaphase FISH evaluation including confirmation and clarification of suspected 
chromosome aberrations or characterization of an apparently abnormal clone. Metaphase cell evaluation may help clarify specific chromosome rearrangements.

e. Fresh-frozen tumor tissues

i. Such tissues may be useful in sequential analysis of recurring tumors or in evaluation of archived samples.

6.7.2.4 Documentation. Analysis and documentation of FISH results should be in accordance with Section E9 of these Standards and Guidelines for Clinical Genetics Laboratories. ${ }^{4}$

\subsubsection{CMA analysis}

6.7.3.1 CMA can provide valuable information to supplement that of chromosomal and FISH analyses. Isolated tumor DNA hybridized to whole-genome copy number and/or singlenucleotide polymorphism microarrays allows detection of loss, gain, and amplification of regions of DNA, which may not otherwise be detected. Single-nucleotide polymorphism probes allow detection of large regions of loss of heterozygosity, which may harbor tumor-suppressor genes. ${ }^{5}$

6.7.3.2 Sample types that may be used for CMA analysis include (i) fresh tumor tissue; (ii) paraffin-embedded tumor tissue; (iii) frozen tumor; and (iv) cultured cells, chromosomally characterized when possible.

a. Fresh tumor tissue

i. If the tumor is homogeneous, fresh tumor is the optimal sample for CMA and can be procured at the time of sample processing for chromosomal analysis. A small piece of identified tumor should be transferred to the microarray laboratory as soon as possible for DNA isolation. For heterogeneous tumors with areas of necrosis, normal tissue, or prominent stoma, DNA isolation from histologically characterized formalinfixed paraffin-embedded material may be needed to ensure that isolated DNA is from the tumor.

b. Paraffin-embedded tumor

i. A pathologist should review the hematoxylin and eosin-stained section of the tumor to identify an area of concentrated tumor for DNA isolation.

c. Fresh-frozen tumor

i. Frozen stored tumor should provide high-quality DNA for CMA. A pathologist's review of the original H\&E-stained slides can assure the frozen sample contains adequate tumor.

d. Cultured tumor cells

i. Tumor cells that have been placed into culture may be used for DNA isolation and CMA as long as they remain viable. An early decision to use cells for CMA is best to minimize growth of normal tissue components.

ii. DNA from cultured and harvested tumor cells that have been chromosomally characterized as abnormal may be used for CMA.
6.7.3.3 Documentation: analysis and documentation of CMA studies should be in accordance with Section E11 of these Standards and Guidelines for Clinical Genetics Laboratories. ${ }^{5}$

\subsection{TURNAROUND TIME AND REPORTING}

\subsubsection{Turnaround time}

6.8.1.1 TAT should be appropriate for clinical utility. The cytogenetics laboratory may want to have a written policy describing how tumor cases are prioritized (with respect to each other and with respect to other sample types) such that the genetic information provided can be used for patient management.

6.8.1.2 TAT guidance:

a. Because of the multiplicity of tumor types and the different tumor growth characteristics in culture, TATs will vary. However, the final report for each tumor should be available as soon as possible given such factors. Final results should be available within 28 calendar days.

b. Tumor FISH analysis results should be available within 1 to 4 days for most tumors and within 7 days for paraffinembedded tumors.

c. Preliminary verbal reports may be appropriate for some case studies. If preliminary results are communicated, then the date of preliminary report should be documented in the final report. The content of the preliminary report should be documented if it differs significantly from that of the final report.

\subsubsection{Reporting}

6.8.2.1 The most recent edition of the International System for Human Cytogenetic Nomenclature should be used to report the chromosomal, FISH, CMA, and sequencing results. ${ }^{6}$

6.8.2.2 Cells analyzed (both normal and abnormal) should be documented in the final report.

6.8.2.3 If an aberration is suspected to be constitutional, analysis of a phytohemagglutinin (PHA)-stimulated blood sample during remission is recommended to clarify the constitutional versus clonal nature of the aberration so genetic counseling may be recommended as appropriate.

6.8.2.4 The final report(s) for tumor samples should contain the following information:

1. Patient identification using two different identifiers

2. Patient medical record number and/or laboratory identification number

3. Name of referring physician

4. Sample information (type, dates of collection and receipt, date of report)

5. Reason for referral or suspected diagnosis

6. International System for Human Cytogenetic Nomenclature of all studies performed

7. Narrative description of the aberrations observed. The report should associate results if more than one study was performed on the same tissue. The interpretation should correlate the genetic testing results with the histopathology report and patient-specific clinical information. 
Discussion can include the clinical significance of the results for the diagnosis, prognosis, and/or therapeutic management of the patient with reference to current literature.

8. Literature references should be included to support the interpretation and to provide helpful information for the health-care provider.

\section{SUPPLEMENTARY MATERIAL}

Supplementary material is linked to the online version of the paper at http://www.nature.com/gim

\section{ACKNOWLEDGMENTS}

The ACMG Working Group acknowledges Marilu Nelson, Laboratory Supervisor at the University of Nebraska Human Genetics Laboratory in Omaha, NE, for her extensive contribution to Supplementary Table S5 online (lymphomas), Felix Mitelman at the University of Lund in Lund, Sweden, for his review and helpful comments on Supplementary Tables S1-5, and Matthew Meredith, postdoctoral fellow at the Harvard Medical School in Boston, MA, for his help with Supplementary Table S2 (genitourinary tumors). These technical standards and guidelines were approved by the ACMG Board of Directors on 25 January 2016.

\section{DISCLOSURE}

All of the authors direct clinical cytogenetics laboratories that run the tests discussed in the current standards and guidelines on a fee-for-service basis.

\section{REFERENCES}

1. Cooley LD and Wilson KS. The cytogenetics of solid tumors. In: Gersen SL and Keagle MB (eds). The Principles of Clinical Cytogenetics 2013. 3rd edn. Springer: New York.

2. Swerdlow SH, Campo E, Harris NL, Jaffe ES, Pileri SA, Stein H, Thiele J, Vardiman JW (eds). WHO Classification of Tumours: WHO Classification of Tumours of Haematopoietic and Lymphoid Tissues 2008. 4th edn. IARC Press: Lyon, France.

3. Zordan A. Fluorescence in situ hybridization on formalin-fixed, paraffinembedded tissue sections. Methods Mol Biol 2011;730:189-202.

4. Mascarello JT, Hirsch B, Kearney HM, et al.; Working Group of the American College of Medical Genetics Laboratory Quality Assurance Committee. Section E9 of the American College of Medical Genetics technical standards and guidelines: fluorescence in situ hybridization. Genet Med 2011;13: 667-675.

5. Cooley LD, Lebo M, Li MM, Slovak ML, Wolff DJ; Working Group of the American College of Medical Genetics and Genomics (ACMG) Laboratory Quality Assurance Committee. American College of Medical Genetics and Genomics technical standards and guidelines: microarray analysis for chromosome abnormalities in neoplastic disorders. Genet Med 2013;15: 484-494.

6. Shaffer LG, McGowan-Jordan J, Schmid M (eds). An International System for Human Cytogenetic Nomenclature (ISCN) 2013. S. Karger: Basel, Switzerland. 